\title{
Bacterial microbiota composition of a common ectoparasite of pÿcavity breeding birds, the Hen Flea Ceratophyllus gallinae
}

\section{Aivelo, Tuomas}

2020-07

Aivelo , T \& Tschirren , B 2020 , ' Bacterial microbiota composition of a common pÿectoparasite of cavity breeding birds, the Hen Flea Ceratophyllus gallinae ' , Ibis , vol. 162 , no. 3 , pp. 1088-1092 . https://doi.org/10.1111/ibi.12811

http://hdl.handle.net/10138/317298

https://doi.org/10.1111/ibi.12811

cc_by_sa

submittedVersion

Downloaded from Helda, University of Helsinki institutional repository.

This is an electronic reprint of the original article.

This reprint may differ from the original in pagination and typographic detail.

Please cite the original version. 
Bacterial microbiota composition of a common ectoparasite of cavity-breeding birds, the Hen Flea Ceratophyllus gallinae

TUOMAS AIVELO ${ }^{1,2} \&$ BARBARA TSCHIRREN ${ }^{3 *}$

${ }^{1}$ Department of Evolutionary Biology and Environmental Studies, University of Zurich, Zurich, Switzerland

${ }^{2}$ Organismal and Evolutionary Biology Research Program, University of Helsinki, Helsinki,

Finland

${ }^{3}$ Centre for Ecology and Conservation, University of Exeter, Treliever Road, Penryn, TR10 9FE, United Kingdom

*Corresponding author.

Email: b.tschirren@exeter.ac.uk 
Hen fleas Ceratophyllus gallinae are common haematophagous ectoparasites of cavity-breeding birds. Experimental field studies have demonstrated negative fitness consequences of hen flea infestations for bird hosts. Yet it is currently unclear if these negative effects are a direct consequence of flea-induced blood loss or a result of flea-borne pathogen transmission. Here we used a 16S rRNA sequencing approach to characterise the bacterial microbiota community of hen fleas collected from Great Tit Parus major nests. We found that Staphylococcus (Firmicutes), Brevibacterium (Actinobacteria), Stenotrophomonas (Proteobacteria), and Massilia (Proteobacteria) and as well as the arthropod endosymbionts Candidatus Lariskella and Candidatus Midichloria were most abundant. We found evidence for the occurrence of Staphylococcus spp. in fleas, which may cause opportunistic infections in bird hosts, but not of known pathogens commonly transmitted by other flea species, such as Bartonella spp. or Rickettsia spp. However, the pathogenic potential of many bacterial groups observed in our study is currently unknown. Furthermore, hen fleas might transmit other pathogens (e.g. viruses) which might contribute to the negative fitness consequences of hen flea infestations for their bird hosts in addition to the direct blood loss or secondary infections of wounds caused by biting fleas.

Keywords: host-parasite interactions, zoonotic diseases, nestboxes, garden birds, next generation sequencing (NGS), microbiome, pathogens, public health, wildlife disease 
The hen flea Ceratophyllus gallinae is a common, generalist ectoparasite of cavity- and semi-cavity breeding birds of the Western Palearctic, such as tits (Paridae), sparrows (Passeridae) or flycatchers (Muscicapidae) (Tripet \& Richner 1997). They live and reproduce in the nesting material and suck blood from nestlings and adults (Tripet \& Richner 1997). Experimental manipulations of hen flea load in great tit nests demonstrated substantial negative fitness consequences of flea infestations for the bird host (e.g. Tschirren et al. 2003, Fitze et al. 2004). Yet, it is currently unknown whether these negative effects are caused directly by the ectoparasite (i.e. through blood loss) or indirectly through the transmission of flea-borne pathogens.

Many members of the flea order Siphonaptera are known vectors for wildlife, pet and / or human pathogens (Bitam et al. 2010). In total, there are over 2500 described flea species; the majority parasitises mammals, but about 6\% are ornithophilic (Bitam et al. 2010). The most famous example of a flea-transmitted pathogen is Yersinia pestis, the causative agent of bubonic plague, which is vectored by a range of mammal flea species, including the Rat Flea Xenopsylla cheopis (Stenseth et al. 2008). Other pathogens transmitted by fleas include Rickettsia spp., the causative agents of diseases such as murine typhus (Traub 1978) and spotted fever (Pérez-Osorio et al. 2008), Bartonella spp., the causative agents of diseases such as cat-scratch disease and endocarditis (Chomel et al. 2006) or Coxiella spp., the causative agents of diseases such as Q fever (Angelakis et al. 2014). The vector potential of bird fleas, on the other hand, is largely unknown. However, a previous study on Ceratophyllus garei fleas infesting migratory reed warblers and their nests in Slovakia observed a Rickettsia spp. prevalence of 31.9\% (Sekeyova et al. 2012), indicating that also bird fleas can act as vectors. Furthermore, evidence of Bartonella spp. infections was found in a range of bird species (Mascarelli et al. 2014).

Here we characterise for the first time the bacterial microbiota of Ceratophyllus gallinae fleas and survey the occurrence of known pathogens, for which hen fleas may act as a vector and which contribute to the lower fitness observed in hen flea-infested birds. 
Understanding the vector-potential of hen fleas is not only important for our understanding of the eco-evolutionary dynamics of bird-ectoparasite interactions, but also for the identification of potential human health risks, as people are exposed to and bitten by hen fleas on a regular basis when cleaning out nest boxes in their garden (du Feu 1987).

\section{Methods and materials}

\section{Flea sampling}

We collected Hen Fleas Ceratophyllus gallinae from 22 nests of Great Tits Parus major breeding in nestboxes in Zurichbergwald, Switzerland $\left(47^{\circ} 20^{\prime} 08^{\prime \prime} \mathrm{N}, 8^{\circ} 30^{\prime} 01^{\prime \prime} \mathrm{E}\right)$ in summer 2016. Fleas were stored in $95 \%$ ethanol.

\section{Bacterial microbiota sequencing}

DNA isolation was performed in a laminar flow cabinet. Individual fleas $(n=208$ fleas from 22 nests; Table 1) were washed thrice with sterile water, once with $3 \%$ hydrogen peroxide and then crushed with a sterile pestle. Fleas from 7-8 nests were then pooled (Table 1) for DNA extraction and $16 \mathrm{~S}$ sequencing. Nests were randomly allocated to pools. Whole-pool DNA extraction was performed using DNeasy Blood \& Tissue kit (Qiagen, Hilden, Germany), following the manufacturer's protocol.

We quantified the bacterial microbiota of hen fleas by sequencing the hypervariable V3-V4 region of the 16S rRNA gene. The protocol has been described in detail elsewhere (Aivelo et al. 2019). In short, we prepared sequencing libraries following the Earth Microbiome 16S Illumina protocol using the primers 515F and 806R (Apprill et al. 2015, Parada et al. 2016), with an expected amplicon size of approximately $300 \mathrm{bp}$. The $16 \mathrm{~S}$ region was amplified in a first PCR reaction, the PCR products were visualized on an agarose gel, cut out and purified with MinElute Gel Extraction kit (Qiagen, Hilden, Germany). Illumina Miseq adaptors were then added in second PCR step and 
the products were purified as described above. $4 \mathrm{nM}$ library pools were then created by mixing equimolar amounts of products, followed by standard normalization protocols. Paired-end sequencing of flea pools and negative controls $(n=5)$ was performed on a Illumina MiSeq at the Functional Genomic Center Zurich using reagent kit 600cycle v3 (Illumina, San Diego, CA, USA) following the manufacturer's protocol, with a target amplicon length of $250 \mathrm{bp}$. Overall, we obtained 918 - 23296 amplicons across flea pools. We analyzed the sequence data with mothur pipeline (Schloss et al. 2009), following MiSeq standard operation procedures (Kozich et al. 2013). For sequence alignment, we used aligned SILVA bacterial references (release 132;

https://www.arbsilva.de/documentation/release-132/) and grouped operational taxonomic units (OTUs) based on 99\% sequence similarity. OTUs were assigned to taxa with the wang method (Wang et al. 2007) using SILVA taxonomy. We used taxonomic lists in Bitam et al. (2010), McElroy et al. (2010) and Eisen \& Gage (2011) to identify flea-borne pathogens among the OTUs. Endosymbionts were identified by a literature search that combined the taxonomic labels and 'endosymbiont' as search terms in Web of Science (Clarivate Analytics, Philadelphia, PA, USA), following Aivelo et al. (2019). Sequences have been deposited to the Sequence Read Archive under BioProject PRJNA528393. Samples were rarefied to lowest amplicon count (1017) to account for amplicon number variation across pools. All OTUs with less than three amplicons across pools were discarded; these OTUs might represent sequencing errors or very rare bacteria that are unlikely to have major effects on host populations. Rarefaction did not alter the OTU composition of the more deeply sequenced pools 1 and 2. Indeed, Bray-Curtis dissimilarities between the nonrarefied and rarefied pool 1 was 0.14 , and 0.09 in pool 2 . The main effect of rarefaction was the removal of rare OTUs (from 954 to 58 in pool 1 and from 463 to 68 in pool 2). To compare the composition of the pools, we calculated alpha diversity (Inverse Simpson Index (Simpson 1949)) and beta diversity (Bray-Curtis dissimilary (Bray \& Curtis 1957) and UniFrac distance (Lozupone et al. 2011)) indices by using vegan (Oksanen et al. 2013) package in R (R Core Team 2013). 


\section{Results}

After filtering, 95 bacterial OTUs were identified across the three flea pools (mean: 66, range 5873; Suppl. Table 1). 28 OTUs were observed in all three pools and 64 OTUs in two of the three pools (Suppl. Table 1). Inverse Simpson index (range: 7.0-11.8; Table 1), Bray-Curtis dissimilarity index (range: 0.49-0.64; Suppl. Table 2) and weighted UniFrac distances (range: 0.39-0.45; Suppl. Table 2) between pools were comparatively uniform, suggesting that there is a moderate level of dissimilarity among pools but none of the pools is markedly different in composition.

Most of the amplicons (97.7\%) belonged to three bacterial phyla: Actinobacteria (24.0\%), Firmicutes (29.2\%) and Proteobacteria (44.4\%)(Fig. 1; Suppl. Table 1). The most common OTUs were Staphylococcus (Firmicutes), Brevibacterium (Actinobacteria), Massilia (Proteobacteria), Stenotrophomonas (Proteobacteria), Sporosarcina (Firmicutes) and (Fig. 1; Suppl. Table 1).

No known bird or mammal pathogens were detected in the sequence data.

However, we found evidence for the occurrence of the arthropod endosymbionts Candidatus Lariskella, Candidatus Midichloria (both among the 6 most common OTUs) and Rickettsiella in hen fleas. No evidence for the occurrence of other arthropod endosymbionts (such as Wolbachia spp. or Candidatus Cardinium) was found.

\section{Discussion}

We characterised the bacterial microbiota of the hen flea C. gallinae, a common, nest-based ectoparasite of cavity-nesting birds, using a 16S sequencing approach. In total, we observed 95 different OTUs across sequencing pools, which is broadly in line with the results of previous 
mammal flea microbiota studies (Pornwiroon et al. 2007, Erickson et al. 2009, Jones et al. 2010, 2015). The phylum Proteobacteria as well as specific bacterial genera, such as Brevibacterium and Stenotrophomonas, were particularly abundant in hen fleas. Interestingly, these groups have also been found to dominate the bacterial communities of mammal flea species (Pornwiroon et al. 2007, Erickson et al. 2009, Jones et al. 2010).

No evidence for the occurrence of flea-transmitted bacterial (bird / mammal) pathogens such as Bartonella spp. or Rickettsia spp. (Bitam et al. 2010, McElroy et al. 2010, Eisen \& Gage 2011) was found in our study. This contrasts the findings of a study in another bird flea, C. garei, which observed a high (31\%) prevalence of Rickettsia spp. (Sekeyova et al. 2012). Currently it is unclear if this difference is due to spatial variation in Rickettsia spp. prevalence (i.e. Slovakia vs Switzerland) or if $C$. gallinae is a non-competent vector. Although studies testing for Rickettsia spp. infections in wild birds are still rare, there is some evidence that infections can occur, but that prevalence is low (Elfving et al. 2010). Similarly, evidence of Bartonella spp. infections has previously been found in several bird species (Mascarelli et al. 2014).

Staphylococcus spp., which can cause opportunistic infections (Coates et al. 2014), was another particularly common OTUs observed in hen fleas. Such opportunistic infections (e.g. of wounds caused by biting fleas) caused by Staphylococcus spp. or other opportunistic pathogens may contribute to the negative fitness effects of hen flea infestations on bird hosts (e.g. Tschirren et al. 2003, Fitze et al. 2004). Other particularly common OTUs across flea pools were arthropod endosymbionts Candidatus_Lariskella and Candidatus_Midichloria. Interestingly, however, we found no evidence for the occurrence of the endosymbiont Wolbachia spp., which is observed in many flea species (Gorham et al. 2006, Jones et al. 2015, Lawrence et al. 2015; but see Jones et al. 2010), including the bird flea C. garei (Sekeyova et al. 2012). It is possible that Wolbachia spp. is not truly absent in C. gallinae but was simply not detected because of low prevalence (only 208 fleas were sequenced in this study). Alternatively, negative interactions among endosymbionts (i.e. 
competition effects; Aivelo et al. 2019) might explain the absence of Wolbachia spp. Indeed, a comparative survey of bacterial microbiota of mammalian fleas suggested that co-occurrence of endosymbionts (such as $C a$. Lariskella, Wolbachia spp., Spiroplasma spp. and Ca. Cardinium) within fleas is rare (Jones et al. 2015). Thus, the comparably high $C a$. Lariskella abundance in hen fleas may impede Wolbachia spp. infections.

Although only three pools of hen fleas were sequenced in our study, they included more than 200 fleas from 22 different great tit nests. Importantly, despite marked among-pool differences for some OTUs, overall the three independent pools showed remarkable similarities in bacterial microbiota composition, with the range of OTU diversity being small and almost half of the OTUs being present in at least two pools. Furthermore, a comparison of non-rarefied and rarefied pools highlighted that the lower sequencing depth of pool 3 did not bias the results. Together, it suggests that the bacterial communities we describe here for $C$. gallinae are representative.

As mentioned above, for some OTU's there were marked among-pool differences, most prominently for Massilia (Proteobacteria), which was very common in pool 1 but rare or absent in the other pools. These differences are likely due to environmental effects (e.g. differences in the composition of the nesting material among nests), which may affect some members of the flea microbiota. Indeed it is typically found that a part of the arthropod microbiota is non-resident (i.e. consists of bacteria that are obtained from the environment) and thus susceptible to environmental influences (Hammer et al. 2017).

Our study has a number of limitations. First, although we did not detect any known bacterial bird or mammal pathogens in our study, we cannot exclude the possibility that hen fleas may act as a vector for them, but that they were not detected because of very low prevalence. Also, for most OTUs observed in our study the pathogenic potential is currently unknown. Furthermore, only hen fleas from great tit nests were analysed and it is possible that hen flea microbiota composition might 
differ depending on host species. Finally, we cannot exclude the possibility that there is spatiotemporal variation in bacterial microbiota composition of hen fleas, or in the occurrence of bacterial pathogens. The characterization of seasonal changes in the microbiota composition of hen fleas feeding on different hosts, as well as variation in hen flea microbiota composition on a large spatial scale would thus be an interesting next step.

In conclusion, we found no evidence that $C$. gallinae fleas harbour known bacterial bird (or mammal) pathogens, such as Rickettsia spp. or Bartonella spp. (Bitam et al. 2010, McElroy et al. 2010, Eisen \& Gage 2011). The negative fitness consequences of hen flea infestations for their bird hosts (Tschirren et al. 2003, Fitze et al. 2004) are thus most likely caused by blood loss, secondary infections of wounds caused by biting fleas and / or other flea-borne pathogens (such as viruses or bacteria that are not currently recognised as bird pathogens).

The lack of (known) human bacterial pathogens in hen fleas furthermore suggests that the human health risk of exposure to hen fleas during nest box cleaning is likely limited. However, the pathogenic potential of most OTUs identified in our study is currently unknown and hen fleas may act as vectors for non-bacterial pathogens. Thus, to prevent exposure to such unidentified flea-borne pathogens, as well as a bad itch, we recommend to clean out nest boxes during a cold winter day when hen fleas are inactive.

\section{Acknowledgments}

This study was funded by Finnish Cultural Foundation Postdoc Pool grant (to TA), the Stiftung für wissenschaftliche Forschung an der Universität Zürich (17_027), the Swiss National Science Foundation (PP00P3_128386 and PP00P3_157455), the University of Zurich Research Priority Program 'Evolution in Action: from Genomes to Ecosystems', and the Faculty of Science of the University of Zurich (all to BT). We thank Lucy Poveda for help with MiSeq sequencing, Frédéric 
Guillaume for providing access to IT infrastructure and anonymous reviewers for constructive comments on the manuscript. 


\section{References}

Aivelo, T., Norberg, A. \& Tschirren, B. 2019. Human pathogen co-occurrence in Ixodes ricinus ticks: effects of landscape topography, climatic factors and microbiota interactions. bioRxiv.

Angelakis, E., Psaroulaki, A., loannou, I., Chochlakis, D. \& Tselentis, Y. 2014. Presence of Coxiella burnetii in Fleas in Cyprus . Vector-Borne Zoonotic Dis. 14: 685-687.

Apprill, A., Mcnally, S., Parsons, R. \& Weber, L. 2015. Minor revision to V4 region SSU rRNA 806R gene primer greatly increases detection of SAR11 bacterioplankton. Aquat. Microb. Ecol. 75: 129-137.

Bitam, I., Dittmar, K., Parola, P., Whiting, M.F. \& Raoult, D. 2010. Fleas and flea-borne diseases. Int. J. Infect. Dis. 14: e667-e676.

Bray, J.R. \& Curtis, J.T. 1957. An ordination of the upland forest community of Southern Wisconsin. Ecol. Monogr. 27: 325-349.

Chomel, B.B., Boulouis, H.J., Maruyama, S. \& Breitschwerdt, E.B. 2006. Bartonella spp. in pets and effect on human health. Emerg. Infect. Dis. 12: 389-394.

du Feu, C.R. 1987. Some observations on fleas emerging from tit nestboxes. Ringing Migr. 8: 123-128.

Eisen, R.J. \& Gage, K.L. 2011. Transmission of Flea-Borne Zoonotic Agents. Annu. Rev. Entomol. 57: 61-82.

Elfving, K., Olsen, B., Bergström, S., Waldenström, J., Lundkvist, Å., Sjöstedt, A., Mejlon, H. \& Nilsson, K. 2010. Dissemination of spotted fever rickettsia agents in Europe by migrating birds. PLoS One 5.

Erickson, D.L., Anderson, N.E., Cromar, L.M. \& Jolley, A. 2009. Bacterial communities associated with flea vectors of plague. J. Med. Entomol. 46: 1532-1536.

Fitze, P.S., Tschirren, B. \& Richner, H. 2004. Life history and fitness consequences of ectoparasites. J. Anim. 
Ecol. 73: 216-226.

Gorham, C.H., Fang, Q.Q. \& Durden, L.A. 2006. Wolbachia Endosymbionts in Fleas (Siphonaptera). J. Parasitol. 89: 283-289.

Hammer, T.J., Janzen, D.H., Hallwachs, W., Jaffe, S.P. \& Fierer, N. 2017. Caterpillars lack a resident gut microbiome. Proc. Natl. Acad. Sci. 114: 9641-9646.

Jones, R.T., Eisen, R., MacMillan, K., Boegler, K. \& Gage, K.L. 2015. Flea-associated bacterial communities across an environmental transect in a plague-endemic region of Uganda. PLoS One 10: e0141057.

Jones, R.T., Knight, R. \& Martin, A.P. 2010. Bacterial communities of disease vectors sampled across time, space, and species. ISME J. 4: 223-231.

Kozich, J.J., Westcott, S.L., Baxter, N.T., Highlander, S.K. \& Schloss, P.D. 2013. Development of a dualindex sequencing strategy and curation pipeline for analyzing amplicon sequence data on the MiSeq Illumina sequencing platform. Appl. Environ. Microbiol. 79: 5112-20.

Lozupone, C., Lladser, M.E., Knights, D., Stombaugh, J. \& Knight, R. 2011. UniFrac: An effective distance metric for microbial community comparison. ISME J. 5: 169-172.

McElroy, K.M., Blagburn, B.L., Breitschwerdt, E.B., Mead, P.S. \& McQuiston, J.H. 2010. Flea-associated zoonotic diseases of cats in the USA: bartonellosis, flea-borne rickettsioses, and plague. Trends Parasitol. 26: 197-204.

Oksanen, J., Blanchet, F.G., Kindt, R., Legendre, P., Minchin, P.R., O’Hara, R.B., Simpson, G.L., Solymos, P., Stevens, M.H.H. \& Wagner, H. 2013. vegan: Community Ecology Package.

Parada, A.E., Needham, D.M. \& Fuhrman, J.A. 2016. Every base matters: Assessing small subunit rRNA primers for marine microbiomes with mock communities, time series and global field samples. Environ. Microbiol. 18: 1403-1414.

Pérez-Osorio, C.E., Zavala-Velázquez, J.E., León, J.J.A. \& Zavala-Castro, J.E. 2008. Rickettsia felis as 
emergent global threat for humans. Emerg. Infect. Dis. 14: 1019-1023.

Pornwiroon, W., Kearney, M.T., Husseneder, C., Foil, L.D. \& Macaluso, K.R. 2007. Comparative microbiota of Rickettsia felis -uninfected and -infected colonized cat fleas, Ctenocephalides felis. ISME J. 1: 394402.

Schloss, P.D., Westcott, S.L., Ryabin, T., Hall, J.R., Hartmann, M., Hollister, E.B., Lesniewski, R. a, Oakley, B.B., Parks, D.H., Robinson, C.J., Sahl, J.W., Stres, B., Thallinger, G.G., Van Horn, D.J. \& Weber, C.F. 2009. Introducing mothur: open-source, platform-independent, community-supported software for describing and comparing microbial communities. Appl. Environ. Microbiol. 75: 7537-41.

Sekeyova, Z., Mediannikov, O., Roux, V., Subramanian, G., Spitalska, E., Kristofik, J., Darolova, A. \& Raoult, D. 2012. Identification of Rickettsia africae and Wolbachia sp . in Ceratophyllus garei fleas from passerine birds migrated from Africa. Vector-borne Zoonotic Dis. 12: 539-543.

Simpson, E.H. 1949. Measurement of diversity.

Stenseth, N.C., Atshabar, B.B., Begon, M., Belmain, S.R., Bertherat, E., Elisabeth, C., Gage, K.L., Leirs, H. \& Rahalison, L. 2008. Plague-past, present and future. Plos Med. 5: e3.

Team, R.C. 2013. R: A language and environment for statistical computing.

Traub, R. 1978. The ecology of murine typhus-a critical review. Trop. Dis. Bullletin 75: 237-317.

Tripet, F. \& Richner, H. 1997. The coevolutionary potential of a 'generalist' parasite, the hen flea $<i>$ Ceratophyllus gallinae<i/>. Parasitology 419-427.

Tschirren, B., Fitze, P.S. \& Richner, H. 2003. Sexual dimorphism in susceptibility to parasites and cellmediated immunity in great tit nestlings. J. Anim. Ecol. 72: 839-845.

Wang, Q., Garrity, G.M., Tiedje, J.M. \& Cole, J.R. 2007. Naive Bayesian classifier for rapid assignment of rRNA sequences into the new bacterial taxonomy. Appl. Environ. Microbiol. 73: 5261-5267. 
Table 1: Number of nests and individual hen fleas included in the sequencing pools and measures of bacterial microbiota diversity in the three pools.

\begin{tabular}{llllll}
\hline Pool & $\begin{array}{l}\text { Number of } \\
\text { nests }\end{array}$ & $\begin{array}{l}\text { Number of } \\
\text { fleas }\end{array}$ & Fleas per nest & Number of & Alpha diversity \\
& & & & OTUs & (Inverse \\
& & & & Simpson index) \\
\hline 1 & 8 & 68 & $8.5(4-11)$ & 58 & 7.0 \\
3 & 7 & $10(2-17)$ & 68 & 11.8 \\
\hline
\end{tabular}




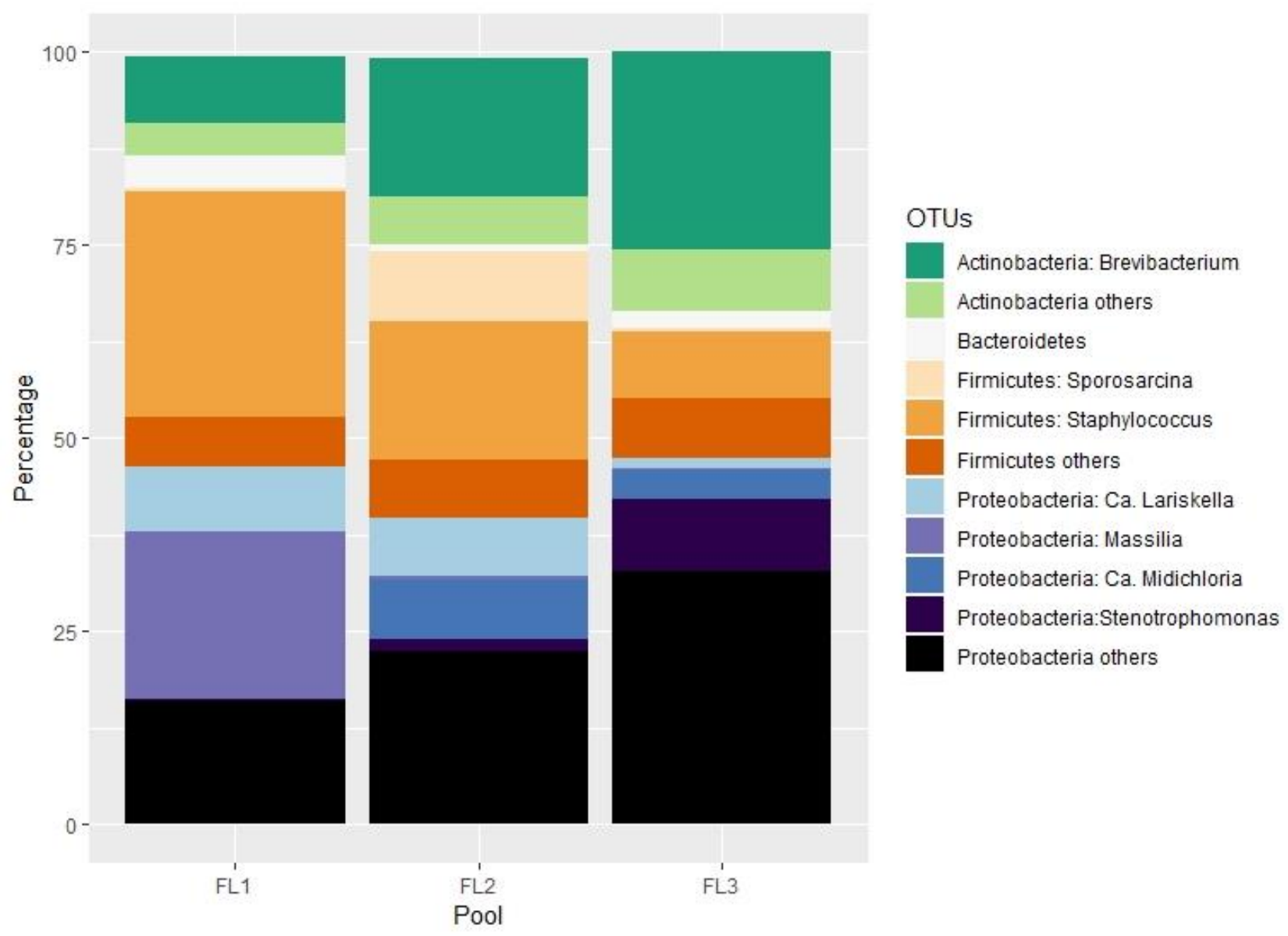

Figure 1: Phylotypic distribution of bacterial OTUs in hen fleas (pools FL1-FL3). The most common OTUs are shown separately whereas less common OTUs are grouped by phyla. 